\title{
Histochemical evaluation of sexual maturation phases in Solea solea wild marine populations
}

\author{
P. R. Werneck*, N. M. M. Lopes*, K. R. De Moraes*, F. Faustino** and F. Morgado* \\ * CESAM \& Departamento de Biologia, Universidade de Aveiro, Campus Universitário de Santiago, \\ 3810-193 Aveiro, Portugal \\ ** Instituto de Ciências Biomédicas Abel Salazar, Universidade do Porto, Portugal
}

The histological analyzes performed in gonad cells to verify the occurrence of changes in their anatomy is of crucial importance in order to characterize the dynamics of the gonads during the sexual maturation phase of its cells in specimens of wild marine populations. These histological data, together with other reproductive data, can be used in the monitoring of marine environments in which the Solea solea (Ishiba et al., 2008) lives, namely, the coastal waters of the Iberian Peninsula, Africa and Mediterranean sea (Ishiba et al., 2008). Therefore, the main objective of this study was to evaluate the several cellular types and its quantities and anatomies (Ishiba et al., 2008). The study was performed using a total of 19 specimens of the species Solea solea, collected between the months of January and March of 2011 in two different locations of the Portuguese coast (Aveiro and Leixões, Portugal). The gonad samples were fixated in Formalin-acetic acid-alcohol (FAA) and processed in accordance to the standard histology protocol (Culling et al., 1985). The slices of the gonads were colored with Haematoxylin and Eosin (H\&E) (used in the study of the general structure of the gonads, as well as in the identification and localization of the different cellular types of the gonad epithelium). As a result of the study, we found 7 different types of gonad cells: I oogonia (It is not photographed ); II a Chromatin nucleolus oocytes (Fig.1. A); II b perinucleolar oocytes (Fig.1. B); III cortical alveoli oocytes (Fig.1. C); IV vitellogenic oocytes (Fig.1. D); V maturing oocyte (Fig.1. E); VI ovulated oocyte (Fig.1. F), (Hibiya, et al., 1995). The different cellular phases were found in all the gonad samples analyzed in this study and the results corresponded to those expected: a decrease in the size in the cells of the first cellular type, the oogonia and an increase in the size in the cells of all other cellular types found in the study. Histological and histochemical analyses of the Solea solea can be used in anatomic and pathological evaluations of the physiological condition of the animals, as stated previously for the muscles, where histological changes could be observed (such as: degeneration and atrophy of the muscle bundles). In the future, the analytical determination of histological and histochemical changes in the gonads of Solea solea, in conjunction with other analytical processes, such as in situ analyzes, can potentially be used to determine and accurately characterize the physiological condition of animals sampled in any natural environment.

\section{References}

1. Hibiya T. and Takashima, F. Atlas of Fish Histology - Normal and Pathological Features. Kodansha, Tókio, Japan, 1995

2. García-lopes Á. et al., Testicular development and plasma sex steroid levels in cultured male Senegalese sole Solea senegalensis Kaup. General and Comparative Endocrinology 147: 343-351, 2006

3. Ishiba R., Quagio-grassiotto I., França G.F., Structural aspects of gonadal development and gonadosomatic ratio of males and females throughout the annual reproductive cycle in Gymnotus cf. Carapo (Teleostei: Gymnotiformes, Gymnotidae)

4. Culling C.F.A., Alison R.T., Barr W.T. (4th ed.), Cellular Pathology technique. London: Butlerworth \& Co., 1985 

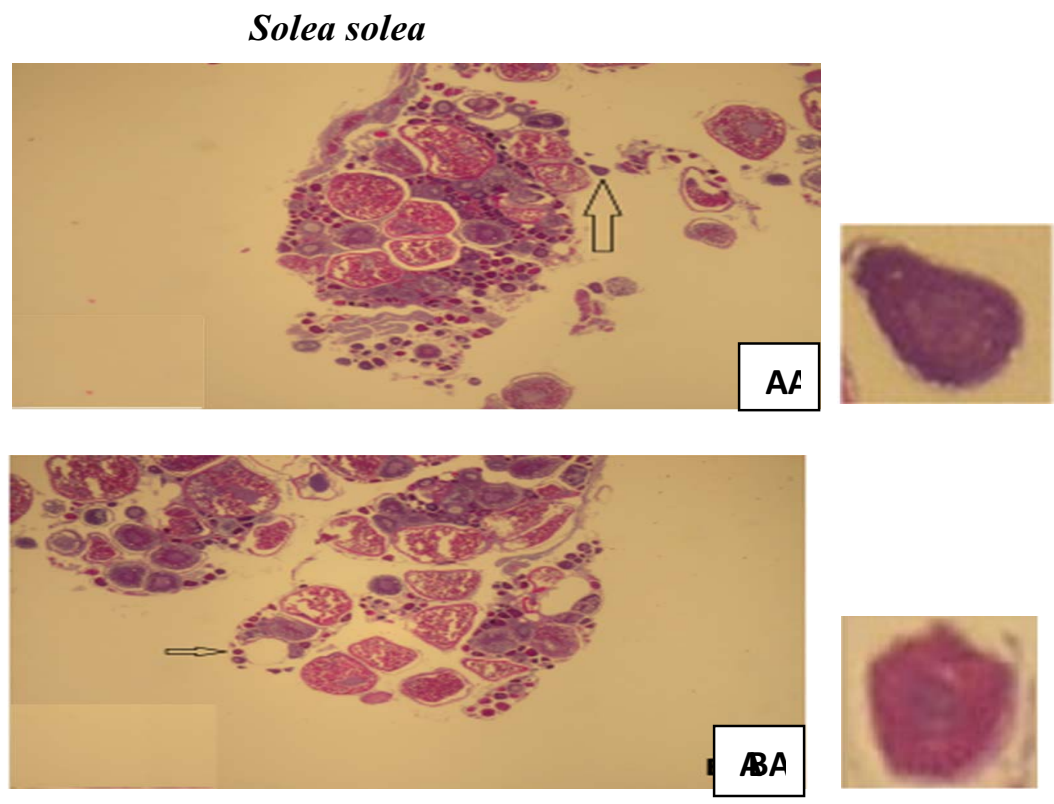

Figure. 1. Light microscopy analysis of H\&E stained gonadal cells of wild marine Solea solea populations, during their sexual maturation phase (scale: 10x). A: arrow indicates the chromatin-nucleolus oocytes. B: arrow indicates perinucleolar oocytes. C: arrow indicates Cortical Alveoli oocytes. D: arrow indicates ovulated oocytes. E: arrow indicates vitellogenic oocyte. $\mathbf{F}$ : arrow indicates maturing oocyte.
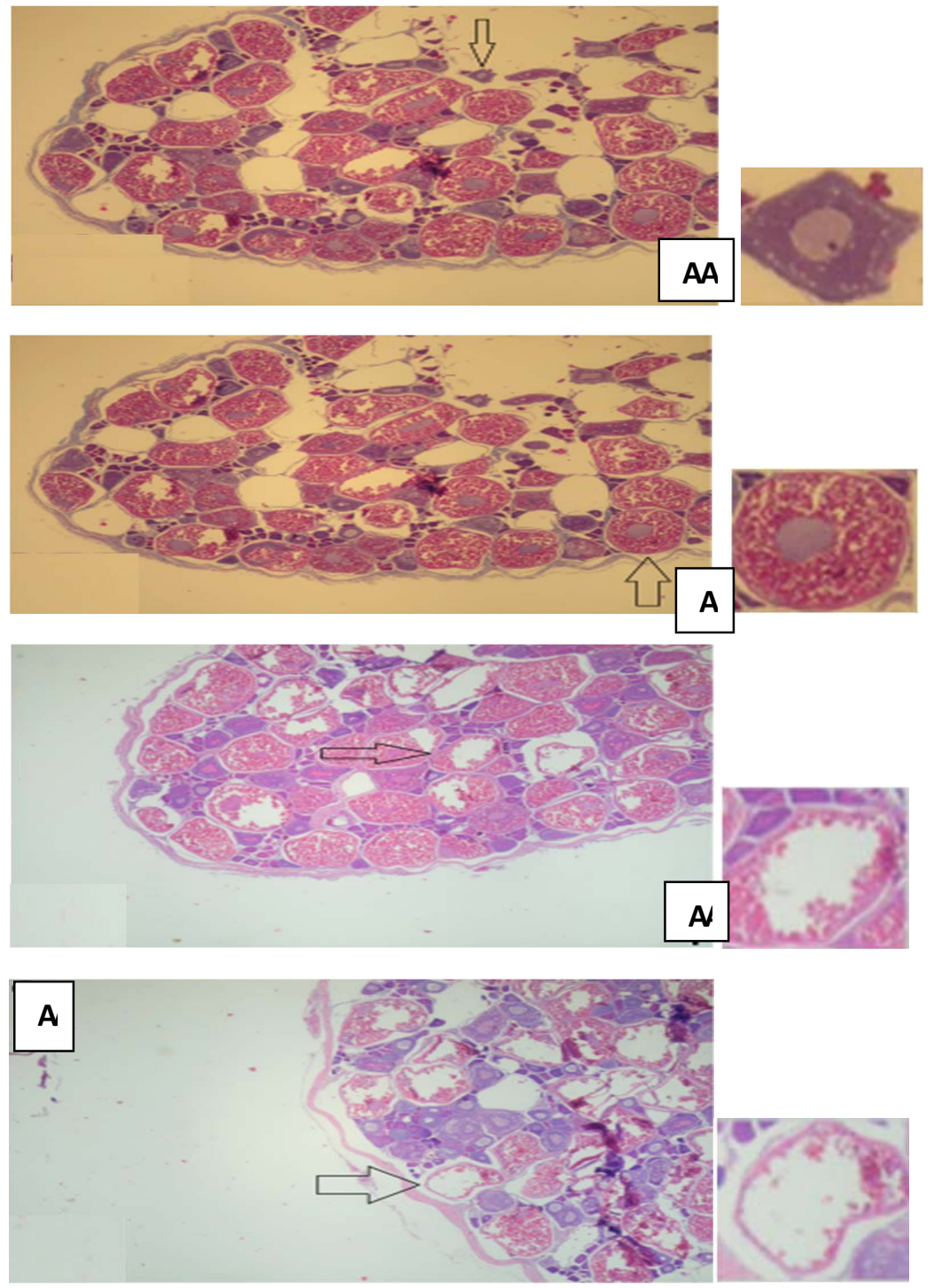